\title{
Dental protection with a padded laryngoscope flange
}

\author{
Christopher L. Pysyk, MD
}

Received: 10 December 2015/Accepted: 14 December 2015/Published online: 21 December 2015

(c) Canadian Anesthesiologists' Society 2015

\section{To the Editor,}

Perioperative dental injury remains a source of concern for patients and anesthesiologists. A recent prospective, observational study of 536 patients assessing dental status before and after tracheal intubation identified enamel fracture as the most common perioperative dental injury. It occurred in $82(15 \%)$ of the 536 patients intubated by direct laryngoscopy. ${ }^{1}$ Not surprisingly, the teeth most likely to experience enamel fracture are the anterior maxillary incisors.

Previously published techniques to mitigate dental injury during laryngoscopy - including the use of dental guards $^{2}$ and covering the laryngoscope flange with compressible, adhesive padding - have been described. ${ }^{3,4}$ A low-cost modification of the latter method of dental protection for patients undergoing laryngoscopy involves application of gauze to the flange of the laryngoscope blade.

The method is simple. Fold one gauze pad into quarters longitudinally (creating a $1 \times 4 \mathrm{~cm}$ pad) (Fig. 1). Then, tape the folded gauze pad onto the flange of the laryngoscope blade - i.e., where it is most likely to interface with the teeth during laryngoscopy (Fig. 2). Thus, a low-profile, non-metallic surface (gauze and tape) has been created with which the enamel of the maxillary teeth may be in contact during laryngoscopy.

Although there are alternative methods of dental protection, such as dental guards, these devices may reduce the amount of oral space available for airway visualization in what could be an already-limited

C. L. Pysyk, MD ( $)$

Department of Anesthesiology, The Ottawa Hospital, University of Ottawa, Ottawa, ON, Canada

e-mail: cpysyk@toh.on.ca oropharynx. The advantage of gauze protection is that it attaches to the flange of the laryngoscope blade such that dental protection is provided where needed without obstructing insertion of the laryngoscope or the laryngoscopic view.

My personal experience with use of the flange-padded laryngoscope blade revealed ease of use and function in a wide range of airways that underwent direct laryngoscopy. Furthermore, the amount of time preparing the

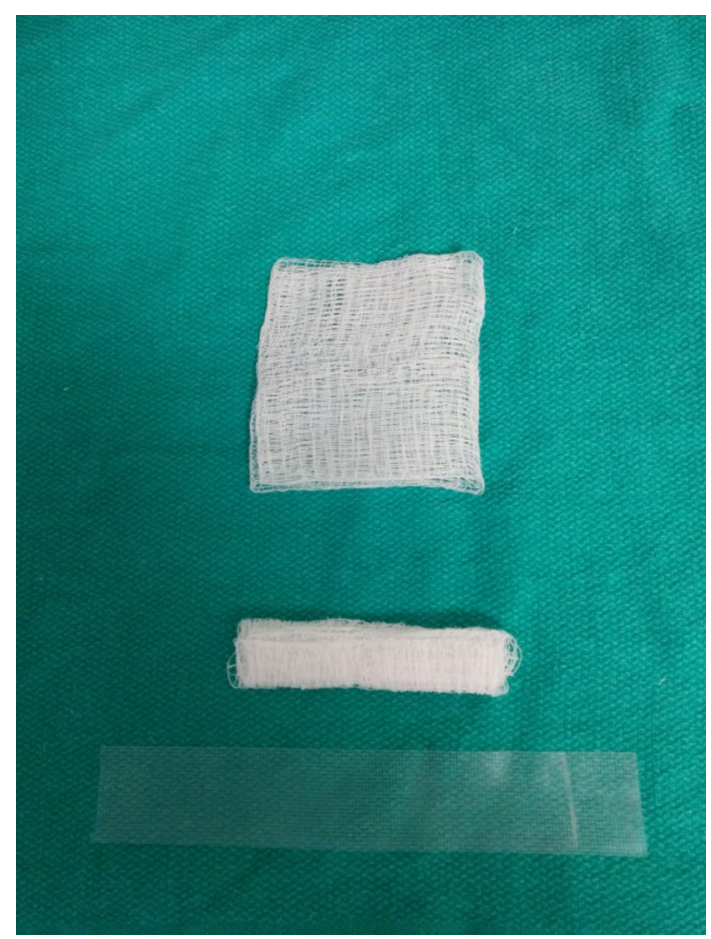

Fig. 1 Gauze unfolded (upper), folded into quarters longitudinally (middle), and plastic tape (bottom) 


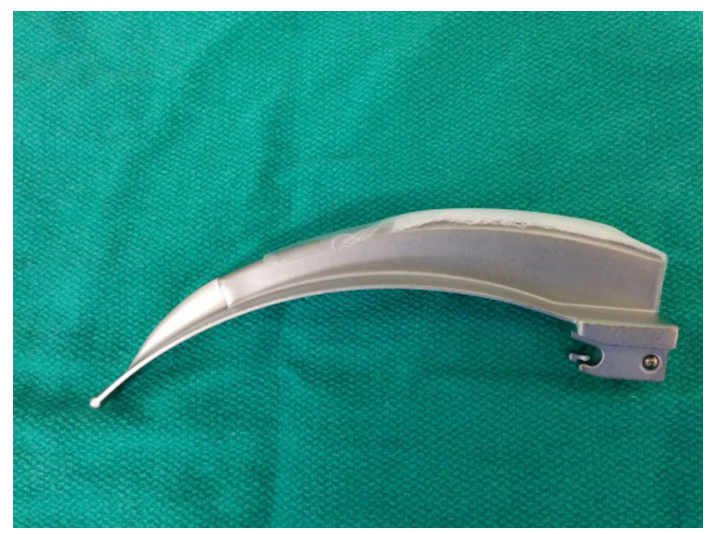

Fig. 2 Gauze pad taped on laryngoscope flange, lateral view

laryngoscope blade does not add significantly to the usual airway equipment preparation.

Although no clinical studies evaluating dental protection with gauze padding on the laryngoscope flange have been reported, the aforementioned technique is a logical, simple, economical, and safe method that requires only readily available perioperative equipment (tape and gauze). Theoretically, it could reduce the likelihood of a common dental injury (e.g., enamel fracture) ${ }^{1}$ during direct laryngoscopy.

Conflicts of interest None declared.

\section{References}

1. Mourao J, Neto J, Luis C, et al. Dental injury after conventional direct laryngoscopy: a prospective observational study. Anaesthesia 2013; 68: 1059-65.

2. Davis FO, DeFreece AB Jr, Shroff PF. Custom-made plastic guards for tooth protection during endoscopy and orotracheal intubation. Anesth Analg 1971; 50: 203-6.

3. Lisman SR, Shepherd NJ, Rosenberg M. A modified laryngoscope blade for dental protection. Anesthesiology 1981; 55: 190.

4. Salcedo E, Modino M (Inventors). Laryngoscope Dental Protection Device. United States patent US 8,235,892 B2. 2012 Aug 7. Available from URL: https://search.rpxcorp.com/pat/8403145/ download (accessed December 2015). 